\title{
Sonda de TDR para estimativa da umidade e condutividade elétrica do solo, com uso de multiplexadores
}

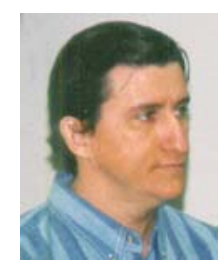

Eugênio F. Coelho ${ }^{1}$, Lucas M. Vellame ${ }^{2}$ \& Maurício A. Coelho Filho ${ }^{1}$

\author{
${ }^{1}$ Embrapa Mandioca e Fruticultura, CP 07, CEP 44380-000, Cruz das Almas, BA. Fone: (75) 6218021. E-mail: \\ ecoelho@cnpmf.embrapa.br (Foto) \\ 2 Escola de Agronomia da UFBA. CEP 44380-000, Cruz das Amas, BA. E-mail: Ivellame@uol.com.br
}

Protocolo 50 - 22/4/2004 - Aprovado em 15/4/2005

\begin{abstract}
Resumo: Com esse trabalho, objetivou-se a caracterização e avaliação de sondas de TDR para determinação da umidade e da condutividade elétrica do solo. As sondas foram construídas para uso direto com a TDR, ou para serem conectadas a multiplexadores, avaliadas para dois tipos de cabo coaxial e para diferentes comprimentos desses cabos (4 a $19 \mathrm{~m}$ ). A constante $\mathrm{K}$ da equação de Giese-Tieman e as impedâncias das sondas foram determinadas e realizadas leituras de diferentes níveis de condutividade elétrica no solo, para diferentes umidades e temperaturas. Verificou-se, comprovada a necessidade do uso de um resistor de $15 \Omega$ em série com a haste central das sondas de TDR para uso com multiplexadores com leitura automática por computador. $\mathrm{O}$ cabo de maior espessura da malha de aterramento e maior diâmetro do condutor interno foi o mais adequado ao uso para as sondas, enquanto o de cabos coaxiais com até $13 \mathrm{~m}$ de comprimento total não afetou significativamente as leituras de umidade; por outro lado, sondas de 0,10 e 0,15 m de comprimento de hastes funcionam com eficiência na medição da condutividade elétrica do solo. A faixa de condutividade elétrica do solo, possível de ser medida com essas sondas, situa-se a até $1,0 \mathrm{dS} \mathrm{m}^{-1}$.
\end{abstract}

Palavras-chave: calibração, reflectometria no domínio do tempo, umidade do solo

\section{TDR probe for estimating soil water content and bulk electrical conductivity by using multiplexers}

\begin{abstract}
This work had as objective the characterization and the evaluation of TDR probes for estimating soil water content and bulk electrical conductivity. The probes were built to be used directly with the TDR 100 or to be connected to multiplexers. The probes were evaluated for two kinds and various lengths $(4$ to $19 \mathrm{~m}$ ) of co-axial cables. The K parameters of Giese-Tieman equation and the probe impedance were determined. The TDR probes were tested for different soil electrical conductivity, under different soil water contents and temperatures. The use of a resistor of $15 \Omega$ connecting the inner wire to the central rod of the TDR probe is necessary for estimating soil water content and bulk electrical conductivity by TDR. The co-axial cable of larger grounding mesh thickness and larger inner wire diameter was most adequate for use with the TDR probes. The use of multiplexers and co-axial cables up to $13 \mathrm{~m}$ length (from TDR to the probe) did not affect significantly water content estimates. The TDR probes of 0.10 and $0.15 \mathrm{~m}$ length performed quite well in estimating soil electrical conductivity up to $1.0 \mathrm{dS} \mathrm{m}^{-1}$.
\end{abstract}

Key words: calibration, time domain reflectometry, soil water content

\section{INTRODUÇÃO}

A TDR (Reflectometria no Domínio do Tempo) vem sendo cada vez mais utilizada para determinação da umidade do solo em pesquisas de manejo e conservação da água e do solo. A determinação indireta da umidade em tempo real por esta técnica é não destrutiva, permitindo a continuidade e automação na coleta dos dados.
Nas pesquisas de fertirrigação, uma área básica a ser considerada se refere à dinâmica de solutos no solo entre fertirrigações, que permite o entendimento dos processos físicos e químicos que ocorrem no volume molhado do solo durante e entre fertirrigações. O maior problema no estudo desses processos reside nos meios disponíveis para coleta de dados que possam inferir o movimento ou a distribuição dos nutrientes. O uso de extratores de solução não permite uma avaliação correta 
da dinâmica dos nutrientes no solo, uma vez que requer determinado tempo para entrada da solução na cápsula, tempo este que varia conforme os teores de água em volta da cápsula porosa, a condutância da cápsula e a diferença entre o potencial de água do solo e dentro da cápsula, após aplicação da sucção.

A utilização da TDR enseja a medição simultânea da umidade volumétrica e condutividade elétrica $(\mathrm{CE})$ do solo em um sistema de coleta não destrutivo, com repetibilidade (Wraith \& Das, 1998).

Diversos tipos de TDR têm sido disponibilizados no mercado e a maioria requer guias de onda para transmitir um pulso eletromagnético, a partir do qual se determina a constante dielétrica aparente do solo (Or \& Wraith, 1997). Os preços dessas guias de onda dos fabricantes, associados aos custos de importação, têm sido limitantes para o uso desses equipamentos.

Várias guias de onda vêm sendo construídas em condições artesanais, com bons resultados (Conciani et al., 1997; Souza et al., 2001; Coelho et al., 2001). Essas guias de onda são muito usadas no Brasil, principalmente com analisadores de umidade do tipo TDR das marcas Tektroniks e Trase System. Um equipamento também disponível é a TDR 100 (Campbell Scientific) que, a exemplo das demais, pode operar com leituras diretas no equipamento ou por meio de multiplexadores de leituras que podem ser eletromecânicos ou eletrônicos. Esses multiplexadores são usados como ferramenta para o aumento dos pontos de medição simultânea de umidade em sistemas automáticos de leitura (Baker \& Almarras, 1990; Coelho \& Or, 1996).

As guias de onda artesanais apresentam, aparentemente, desempenho semelhante em funcionalidade quando acopladas diretamente ao equipamento TDR 100; entretanto, quando acopladas a canais de multiplexadores eletromecânicos, conectados à TDR 100, podem apresentar limitações devido à conexão das guias de onda nesses multiplexadores. Nas conexões ocorre aumento da impedância do sinal, provocando picos de reflexão que impedem as leituras automáticas feitas pelo aplicativo computacional. São necessários ajustes nessas sondas não-industriais para uso com a TDR 100, de forma que possam operar tanto acopladas diretamente ao analisador de umidade como a canais de multiplexadores, fornecendo valores de umidade e de condutividade elétrica aparente do solo.

O objetivo primeiro com este trabalho, foi caracterizar e avaliar uma guia de onda de baixo custo, construída em laboratório para leituras de umidade e de condutividade elétrica, quando conectadas diretamente à TDR 100 ou a um multiplexador eletromecânico.

\section{MATERIAL E MÉTODOS}

As sondas de TDR (guias de onda) foram construídas para atender ao equipamento de TDR 100, da Campbell Scientific, e serem acopladas diretamente ao equipamento ou a um conjunto de multiplexadores de leituras do tipo SMDX, da mesma empresa. As sondas se compunham de três hastes de 0,15 e $0,10 \mathrm{~m}$, espaçadas $0,022 \mathrm{~m}$, à semelhança das construídas para atender aos equipamentos Tektroniks ou Trase System (Coelho et al., 2001; Souza et al., 2001), com inclusão de um resistor de $15 \Omega$, conectando em série a haste central ao terminal positivo do cabo coaxial (Figura 1) indispensável para o seu funcionamento quando acopladas aos multiplexadores de leitura.

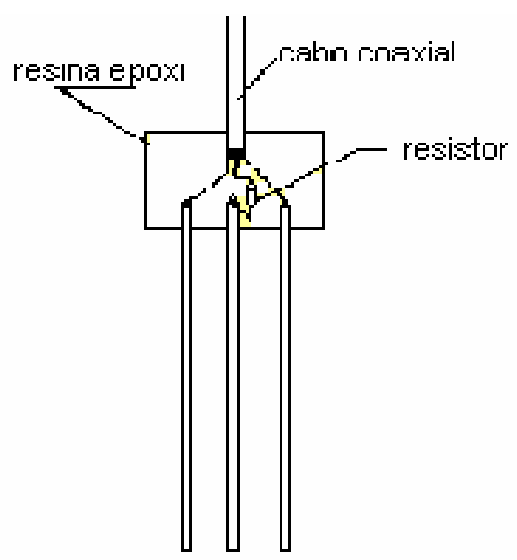

Figura 1. Guia de onda de uso para leitura direta da TDR ou por meio de multiplexadores de leituras

As sondas de hastes de $0,15 \mathrm{~m}$ de comprimento foram avaliadas com dois cabos coaxiais diferentes para se verificar o efeito dos mesmos nas medidas de umidade. O cabo 1 (Cb1) apresentou malha de aterramento com fio de $0,00012 \mathrm{~m}$ de espessura e $0,72 \mathrm{~m}$ de fio por $\mathrm{cm}$ de comprimento do cabo e espessura do condutor central de $0,00083 \mathrm{~m}$. O cabo $2(\mathrm{Cb} 2)$ apresentou malha de aterramento com fio de mesmo diâmetro e com uma densidade de $0,24 \mathrm{~m}$ de fio por $\mathrm{cm}$ de comprimento e espessura do condutor central de $0,00056 \mathrm{~m}$. Os cabos foram testados a partir de leituras de umidade e comportamento do sinal eletromagnético diretamente acoplados à TDR ou a canais de um multiplexador (Figura 2).
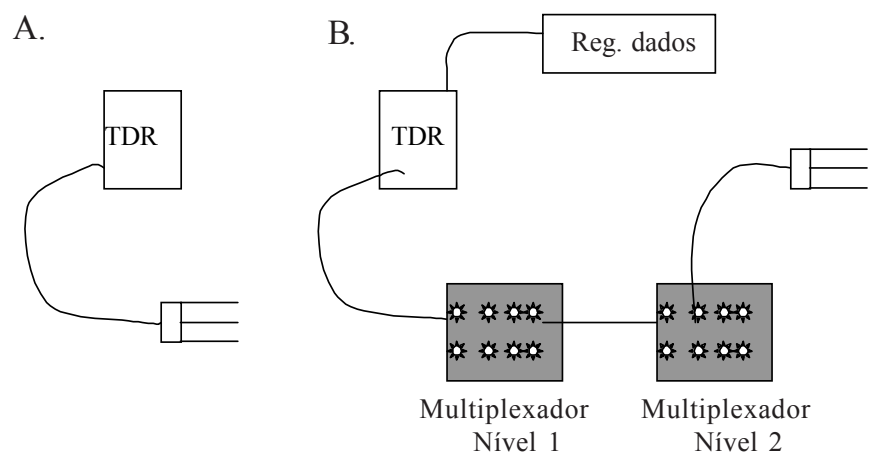

Figura 2. Formas de ligação da sonda à TDR, (A) direta e (B) via multiplexadores

Uma vez definido o cabo mais adequado, avaliou-se o efeito do seu comprimento na forma do sinal, principalmente nas reflexões no início e no final das hastes, (Figura 1), por meio de uma guia de onda com comprimento fixo de $2 \mathrm{~m}$. O comprimento do cabo entre a TDR 100 e o multiplexador de nível zero, foi de $1 \mathrm{~m}$; do nível zero para o nível 1 foi de 4,0;4,5;6,0;8,0;13,0 e $16,0 \mathrm{~m}$. As leituras foram feitas por meio do aplicativo "PCTDR", usando-se a água de torneira e um solo de textura argilosa, como meios para leitura. 


\section{Avaliação da sonda para determinação da condutividade elétrica}

Fez-se a determinação de K (Eq. 1) para as sondas, por imersão das mesmas em soluções (Noborio, 2001) de 160, 420, $510,710,850$ e $920 \mathrm{mg} \mathrm{KCl} \mathrm{L}^{-1}$ e as estimativas da condutividade elétrica pela TDR foram configuradas $\operatorname{com} \mathrm{K}=1$. As condutividades elétricas (CE) das soluções foram medidas por um condutivímetro de bancada. Com os dados da CE medidos das soluções e com as leituras da TDR para $K=1$, referente aos valores de $\left[\left(1 / Z_{u}\right) .\left(1-\rho_{\infty}\right) /\left(1+\rho_{\infty}\right)\right]$, por regressão linear, determinou-se o valor de $\mathrm{K}$ (coeficiente angular da regressão).

Obteve-se o valor da impedância das guias de onda $\left(Z_{\mathrm{o}}\right)$ em $\Omega$ através do $\mathrm{K}$, pela equação:

$$
\mathrm{Zo}=120 \pi \mathrm{LK}
$$

em que:

$\mathrm{L}$ - comprimento das hastes em $\mathrm{m}$

Quatro colunas de PVC foram preenchidas com solo seco passado em peneira de $2 \mathrm{~mm}$ de modo a resultarem em uma densidade aparente de $1,4 \mathrm{~kg} \mathrm{dm}^{-3}$. Em cada coluna de solo foi aplicada uma solução com condutividade elétrica diferente, mantendo-se a umidade volumétrica em 100\% da água disponível do solo. As soluções aplicadas foram de 0,3, 0,5, 0,7 e $1,0 \mathrm{dS} \mathrm{m}^{-1}$. Medições de umidade e condutividade elétrica foram efetuadas pelas guias de onda de 0,10 e $0,15 \mathrm{~m}$ de comprimento de haste.

\section{Efeito da temperatura na condutividade elétrica}

Foram montadas três colunas de solo $\left(795 \times 10^{-6} \mathrm{~m}^{3}\right)$ de textura argilosa e umidades diferentes, aproximadamente 0,37, 0,31 e $0,24 \mathrm{~m}^{3} \mathrm{~m}^{-3}\left(\mathrm{w}_{1}, \mathrm{w}_{2} \mathrm{e} \mathrm{w}_{3}\right)$. Em cada coluna foi inserido um termopar tipo cobre-constantan, ligado a um registrador de dados (CR10X, Campbell Scientific) e uma guia de onda acoplada à TDR 100, por meio de um multiplexador do tipo SMDX50 (Campbell Scientific). As médias de temperatura, umidade e condutividade elétrica do solo, foram coletadas simultaneamente pelo sistema de aquisição de dados (registrador de dados) e armazenadas a cada 2 min.

Iniciou-se o experimento após o aquecimento do solo próximo de $60{ }^{\circ} \mathrm{C}$ de cada coluna, devidamente protegido para evitar a evaporação da água, em estufa. Procedeu-se às leituras das variáveis estudadas, até a temperatura do solo atingir $25^{\circ} \mathrm{C}$; posteriormente, as colunas foram resfriadas em um refrigerador, por $24 \mathrm{~h}$, reduzindo a temperatura para $5^{\circ} \mathrm{C}$, quando se iniciou, então, um novo período de medidas, até a temperatura do solo se igualar à do ambiente $\left(25^{\circ} \mathrm{C}\right)$.

\section{RESULTADOS E DISCUSSÃO}

\section{Avaliação da sonda de TDR para umidade do solo}

A Figura 3 ilustra o sinal do pulso eletromagnético ao longo da guia de onda acoplada diretamente à TDR e ao multiplexador de nível 1 . O uso do resistor de $15 \Omega$ em série aumenta a impedância na zona de conexão do cabo coaxial com as hastes aumentando coeficiente de reflexão no início da haste, em nove vezes o valor gerado sem o resistor (Figura 3A). Quando a guia de onda sem o resistor foi acoplada diretamente a TDR, a reflexão do sinal no começo da sonda não foi detectada pelo aplicativo, no caso da leitura automática, (Figura 3A), visto que pequenos picos no sinal, equivalentes ao acoplamento do cabo no canal do multiplexador, foram suficientes para afetar as leituras de umidade, com falhas do aplicativo CTDR", na identificação do início das sondas. Em se tratando do uso do multi-plexador (Figura 3B), e mesmo com a presença de picos ou reflexões do sinal no acoplamento da guia de onda ao canal do multiplexador, o pico referente ao início da sonda com resistor permitiu ao aplicativo a sua identificação e a leitura da umidade pelo aplicativo.
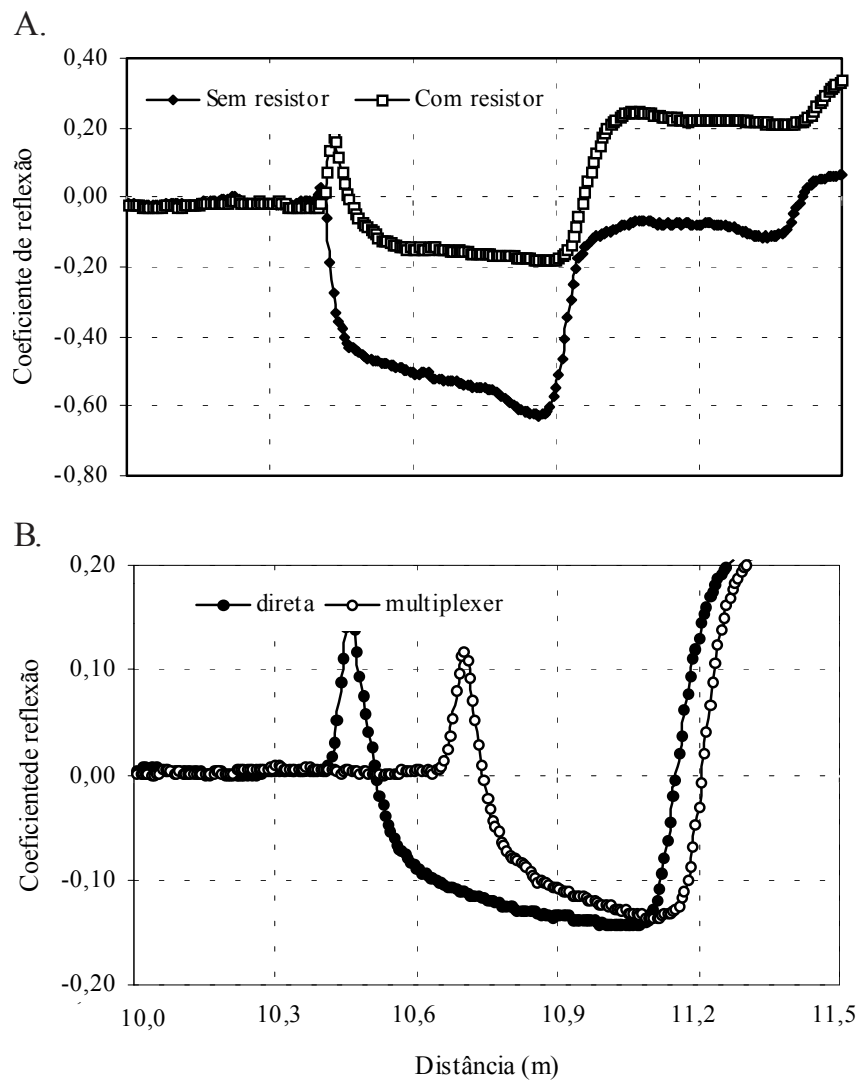

Figura 3. Sinal referente ao pulso eletromagnético emitido pela TDR para a guia de onda com um resistor de $15 \mathrm{ohm}$ e sem resistor (A). Sinal obtido com hastes com resistor, acoplada diretamente à TDR e ao multiplexador (B)

\section{Efeito do tipo e do comprimento do cabo coaxial}

A avaliação do tipo de cabo pode ser vista nas Figuras 4A e B, em que o uso do cabo com menor espessura de malha de aterramento e menor diâmetro do condutor central $(\mathrm{Cb} 2)$, apesar da impedância nominal de $50 \Omega$, apresenta coeficiente de reflexão positivo e relativamente elevado, antes mesmo do início da guia de onda, indicando impedância do cabo, superior à nominal. $\mathrm{O}$ sinal desta forma dificultou, ou inviabilizou, as leituras, principalmente com o uso do multiplexador de leitura (Figura 4B). Os cabos de maior espessura da malha de aterramento e maior diâmetro do condutor interno (Cb1) apresentaram comportamento satisfatório do sinal 
A.

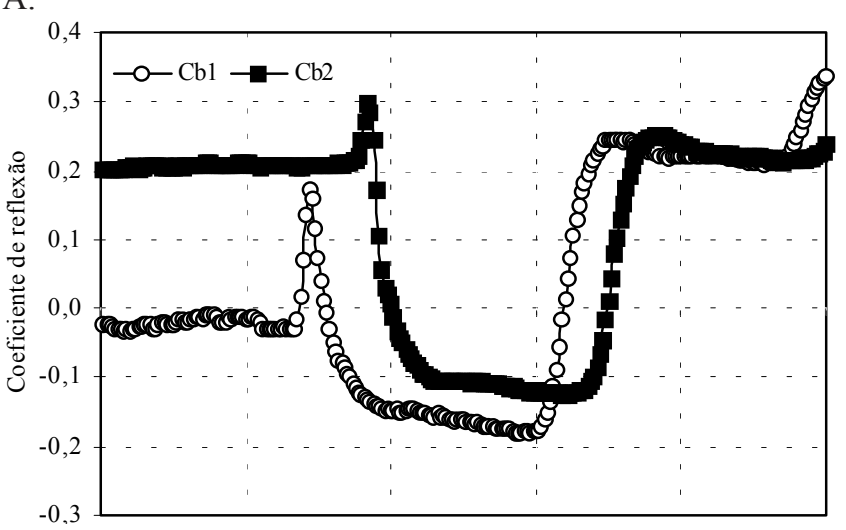

B.

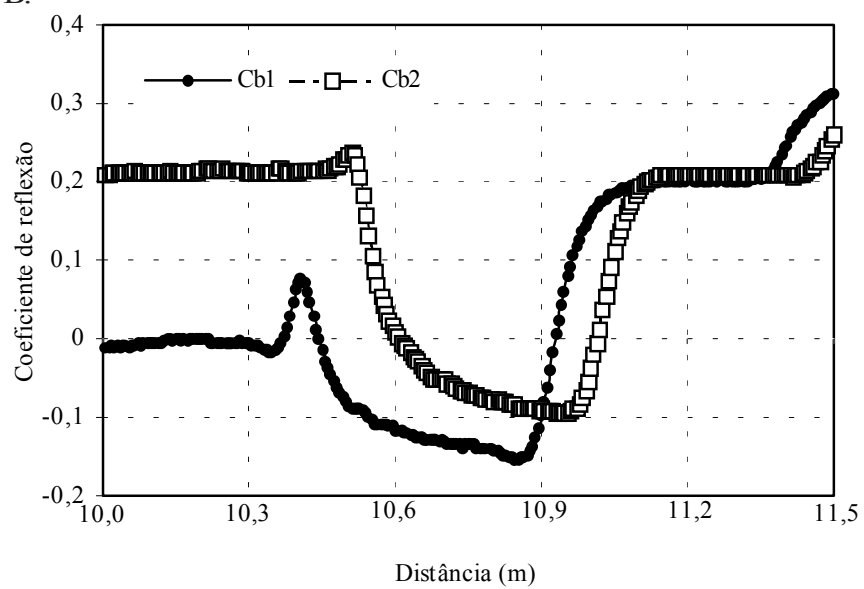

Figura 4. Sinal referente ao pulso eletromagnético emitido pela TDR para a guia de onda acoplada diretamente na TDR 100 (A) e no multiplexador (B)

eletromagnético no visor, sendo adequado para uso como componente de guias de onda.

Vê-se, na Figura 5, as cinco formas de onda resultantes de medições de umidade. (Tabela 1), quando se variou o comprimento dos cabos de ligação do multiplexador de nível zero com o multiplexador de nível 1. Observou-se, também, tendência de atenuação na amplitude do sinal, usando-se cabos de maiores comprimentos e, pelos valores de umidade na Tabela 1, que não houve efeito do comprimento do cabo nas leituras de umidade do solo para comprimento total (TDR - guia de onda) de até $13 \mathrm{~m}$. A variação na média dos valores de umidade foi inferior a $0,75 \%$, quando determinados com cabos de comprimento de até $13 \mathrm{~m}$, tanto no solo como na água. A variação entre a média da umidade do solo, considerando-se todos os

Tabela 1. Umidade volumétrica com a sonda acoplada no multiplexador, para diferentes comprimentos de cabo coaxial

\begin{tabular}{ccc}
\hline \multirow{2}{*}{ Comprimento total do cabo $(\mathrm{m})$} & \multicolumn{2}{c}{ Umidade volumétrica $\left(\mathrm{m}^{3} \mathrm{~m}^{-3}\right)$} \\
\cline { 2 - 3 } & Água & Solo \\
\hline 4,0 & 0,879 & 0,213 \\
4,5 & 0,878 & 0,213 \\
6,0 & 0,856 & 0,217 \\
8,0 & 0,891 & 0,210 \\
13,0 & 0,909 & 0,219 \\
19,0 & 0,931 & 0,237 \\
\hline
\end{tabular}

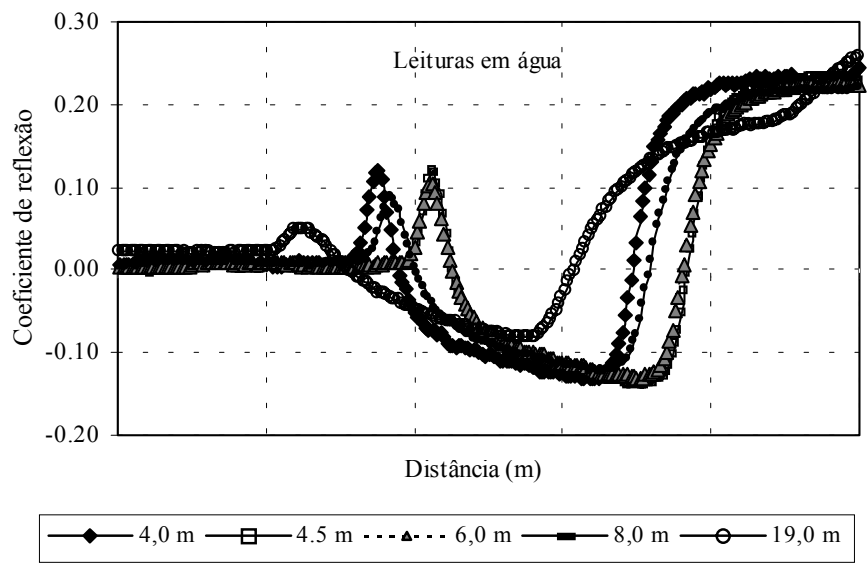

Figura 5. Sinal referente ao pulso eletromagnético emitido pela TDR para a guias de onda com resistor de $15 \Omega$, para cada distância adotada entre a TDR 100 e a guia de onda $(4,6,8$ e $19 \mathrm{~m})$

comprimentos de cabo, inclusive o de $19 \mathrm{~m}$, foi $1,7 \%$ superior à média das umidades, para todos os comprimentos de cabo de até $13 \mathrm{~m}$. Desta forma, comprimentos de cabo, do reflectômetro (TDR) ao multiplexador, de até $14 \mathrm{~m}$, não têm efeito na determinação de umidade, com uso das sondas de TDR de $2 \mathrm{~m}$ de comprimento de cabo e com resistor de $15 \Omega$ no início das hastes.

\section{Efeito da temperatura na determinação da umidade do solo pela sonda de TDR}

Observou-se grande consistência dos valores estimados pelas guias de onda, que apresentaram baixa variabilidade em toda a faixa de temperatura trabalhada, conforme os baixos valores de coeficiente de variação para as três umidades estudadas $\left(\mathrm{w}_{1}, \mathrm{w}_{2}\right.$ e $\left.\mathrm{w}_{3}\right)$ neste caso, (Tabela 2). Observa-se, também, uma ligeira diminuição do coeficiente de variação com o aumento da umidade do solo. A baixa variabilidade pode ser constatada na Tabela 2 , pela reduzida amplitude total dos dados, em média de $0,02 \mathrm{~m}^{3} \mathrm{~m}^{-3}$ da umidade do solo e baixa amplitude interquartil, revelando que $50 \%$ das estimativas foram ainda mais próximos $\left(0,005 \mathrm{~m}^{3} \mathrm{~m}^{-3}\right)$ da umidade (Tabela 2$)$.

A Figura 6 mostra o comportamento geral da umidade, estimada em função da variação da temperatura do solo,

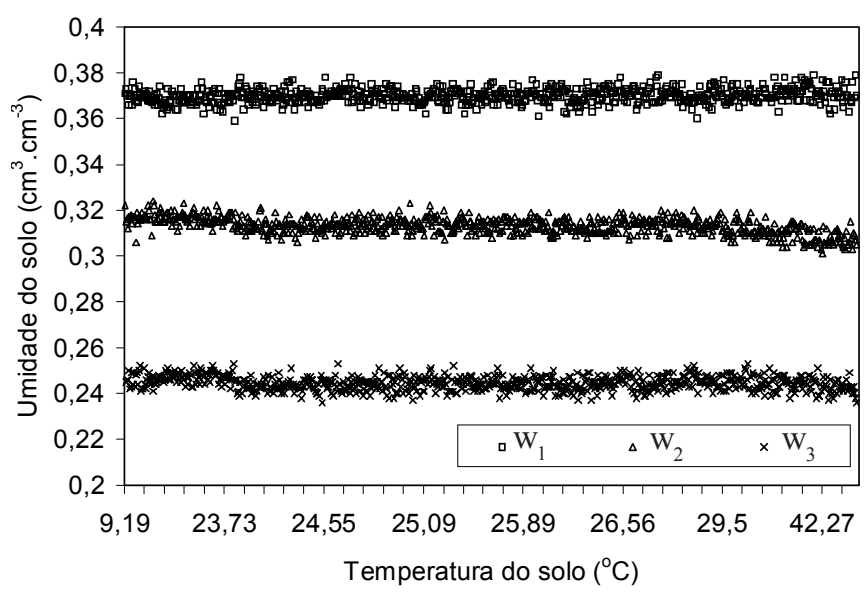

Figura 6. Variação da umidade estimada por guias de onda (TDR 100) com a temperatura do solo 
Tabela 2. Análise descritiva da umidade do solo $\left(\mathrm{m}^{3} \mathrm{~m}^{-3}\right)$ de cada coluna $\left(\mathrm{w}_{1}, \mathrm{w}_{2} \mathrm{e} \mathrm{w}_{3}\right)$ estimada pelas guias de onda construídas para TDR 100

\begin{tabular}{cccccccccc}
\hline & $\begin{array}{c}\text { Número de } \\
\text { observações }\end{array}$ & Média & Mediana & Min. & Max. & $\begin{array}{c}\text { Amplitude } \\
\text { total }\end{array}$ & $\begin{array}{c}\text { Amplitude } \\
\text { interquartil }\end{array}$ & Desvio padrão & CV \\
\hline $\mathrm{w}_{1}$ & 717 & 0,370 & 0,370 & 0,359 & 0,379 & 0,020 & 0,004 & 0,003 & 0,86 \\
$\mathrm{w}_{2}$ & 735 & 0,313 & 0,313 & 0,301 & 0,324 & 0,023 & 0,005 & 0,004 & 1,16 \\
$\mathrm{w}_{3}$ & 734 & 0,245 & 0,245 & 0,236 & 0,253 & 0,017 & 0,005 & 0,003 & 1,27 \\
\hline
\end{tabular}

corroborando o que já foi discutido, concordando com Topp et al. (1980) e não se observando as tendências encontradas por Pepin et al. (1993), Wraith \& Or (1999) e Or \& Jones (2001). Esses autores têm observado, em geral, redução na constante dielétrica do solo, com o aumento de temperatura para condições de maior umidade e menor efeito da temperatura, para condições mais secas do solo, devido ao fato de as moléculas de água se encontrarem mais presas ao solo a baixos níveis de umidade.

\section{Determinação da condutividade elétrica}

As Figuras 7A e B apresentam os valores de $\mathrm{K}$ determinados para as sondas de TDR, em que as sondas de $0,10 \mathrm{~m}$ resultaram

A.

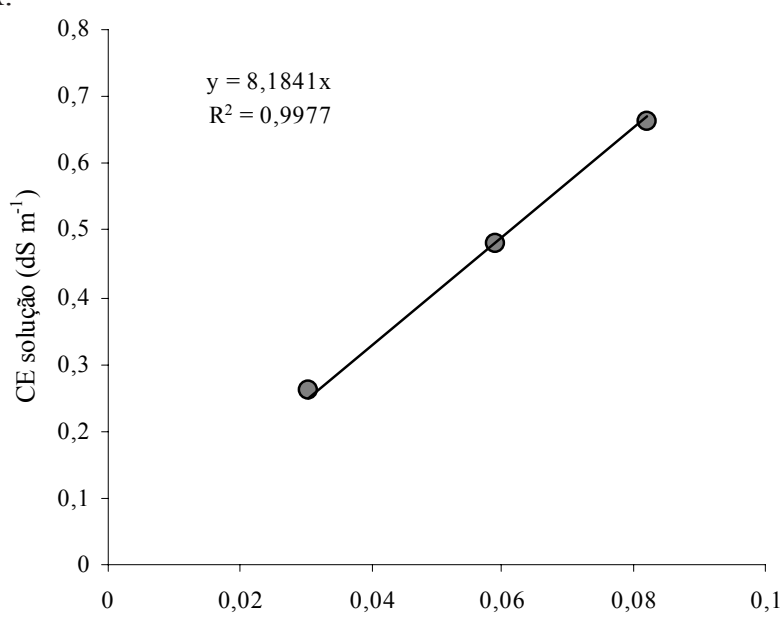

B.

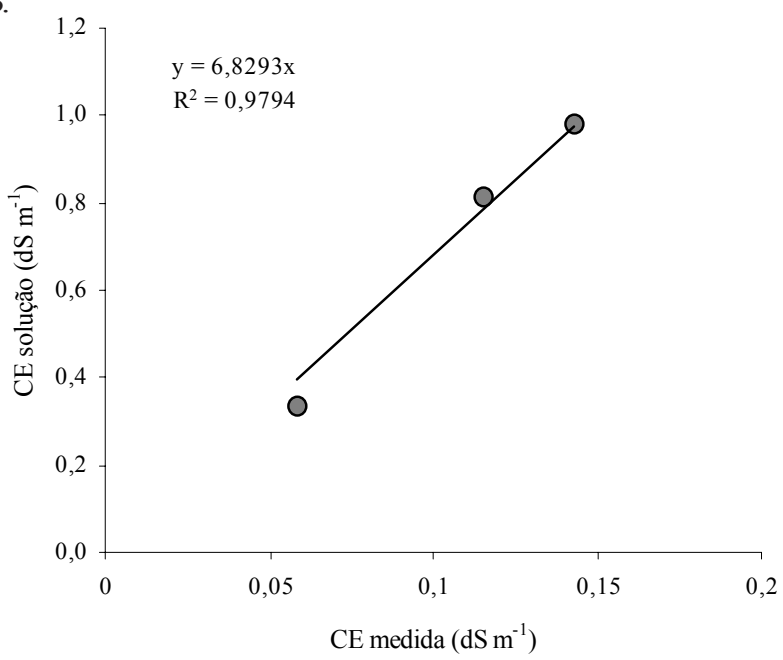

Figura 7. Determinação das constantes geométricas das guias de onda de TDR (K) com 0,10 m (A) e 0,15 m (B) de comprimento de hastes em maior $\mathrm{K}(8,18)$ que a de $0,15(6,83)$. As leituras da condutividade elétrica (CE) pela TDR foram possíveis, porém limitadas a soluções com CE menor que $1,0 \mathrm{dS} \mathrm{m}^{-1}$. Para valores de condutividade elétrica superiores a este, o equipamento acusou erro de leitura. As impedâncias das guias de onda de 0,10 e $0,15 \mathrm{~m}$ foram de 308,53 e $386,19 \Omega$, respectivamente, valores estes superiores aos obtidos pelas guias de onda construídas da mesma forma e sem os resistores de $15 \Omega$ (Silva et al., 2002).

As formas de onda apresentadas nas medições de condutividade elétrica no solo estão ilustradas nas Figuras 8A e $\mathrm{B}$, na qual se observa diminuição do valor de $\mathrm{V}_{\infty}$ na medida em que se aumenta o valor da condutividade elétrica.

Observou-se, em ambas as guias de onda, um valor de $\mathrm{V}_{1}$ elevado, o que se deveu à alta impedância no início da guia de onda devido à inserção do resistor em série, o que promoveu maior reflexão.

\section{Efeito da temperatura na condutividade elétrica}

Os resultados referentes à condutividade elétrica do solo indicaram, como esperado, uma relação linear positiva com a

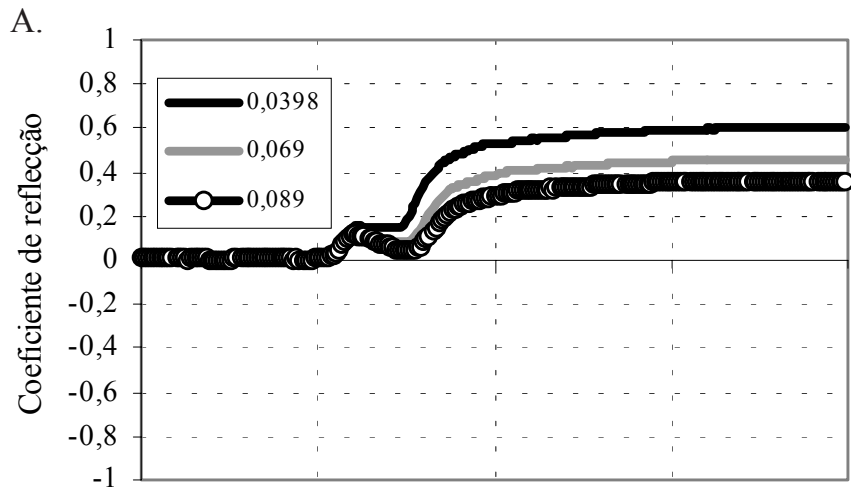

B.

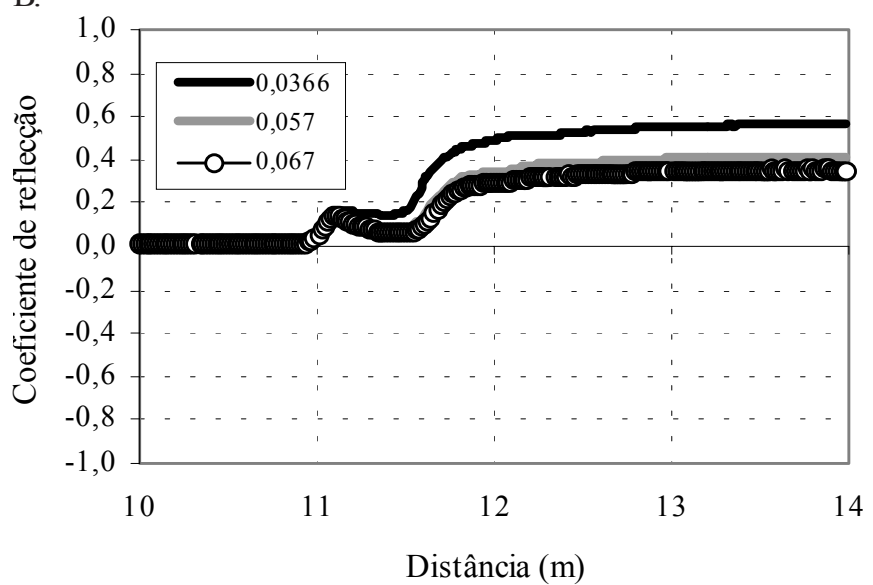

Figura 8. Formas de onda apresentadas pela TDR com o uso de guias com $0,10(\mathrm{~A})$ e $0,15 \mathrm{~m}(\mathrm{~B})$ de comprimento das hastes 
temperatura (Figura 9) e constatou-se que, para os três níveis de umidade, a taxa média de elevação nos valores de condutividade elétrica em função da temperatura, foi de 0,0125 $\mathrm{dS} \mathrm{m}{ }^{-1} \mathrm{C}^{-1}$ para $\mathrm{w}_{1}, 0,0074 \mathrm{dS} \mathrm{m}^{-1}{ }^{\circ} \mathrm{C}^{-1}$ para w2 e $0,005 \mathrm{dS} \mathrm{m}^{-1} \mathrm{C}^{-1}$ para $\mathrm{w}_{3}$, o que mostra maior sensibilidade da $\mathrm{CE}$, a temperatura para maiores níveis de umidade.

Os ajustes lineares dos dados de CE para diferentes temperaturas do solo não concordaram com Hasted (1973) que encontrou ajuste polinomial cúbico para os dados.

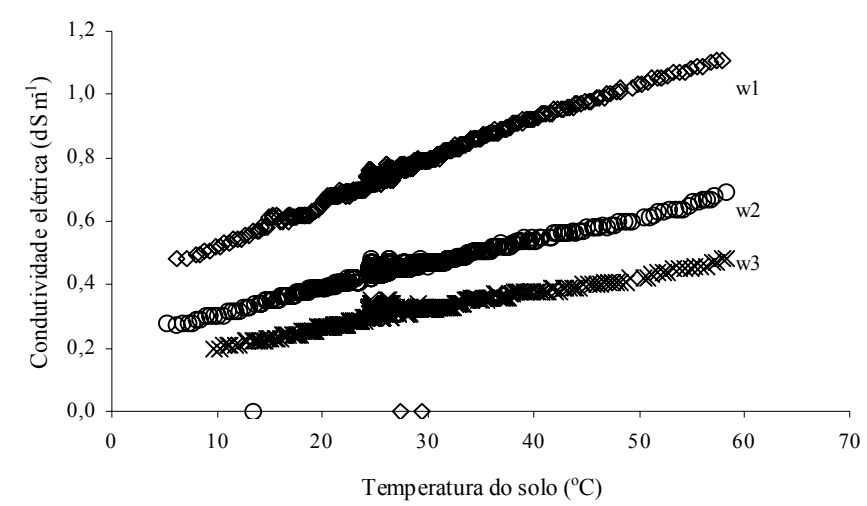

Figura 9. Variação da condutividade elétrica da solução do solo estimadas por guias de onda (TDR 100) em função da variação da temperatura do solo, para os três níveis de umidade estudados $\left(\mathrm{w}_{1}, \mathrm{w}_{2} \mathrm{e}_{3}\right)$

\section{CONCLUSÕES}

1. Para se efetuar leituras de umidade volumétrica na TDR 100 com ou sem multiplexadores de leitura, é necessário o uso de um resistor de $15 \Omega$ em série com a haste central nas guias de onda.

2. As leituras de umidade não foram afetadas para comprimento de cabos coaxiais entre o reflectômetro e multiplexadores de nível zero e 1 de até $13 \mathrm{~m}$, para comprimento do cabo da guia de onda de $1 \mathrm{~m}$.

3. As guias de onda de $0,10 \mathrm{~m}$ e $0,15 \mathrm{~m}$ de comprimento de hastes com resistor de $15 \Omega$ em série com a haste central, funcionam eficientemente na medição da condutividade elétrica do solo, pela TDR 100.

4. A faixa de salinidade do solo possível de ser medida com as guias de onda com resistor de $15 \Omega$, situa-se até $1,0 \mathrm{dS} \mathrm{m}^{-1}$.

5. A condutividade elétrica estimada pela TDR variou linear e positivamente com a temperatura, sendo que suas variações foram mais sensíveis às variações da temperatura, para maiores valores de umidade.

\section{LITERATURA CITADA}

Baker, J.M.; Allmarras, R.R. System for automating and multiplexing soil moisture measurement by Time Domain Reflectometry. Soil Science Society of America Journal, Madison, v.54, n.1, p.1-6. 1990.
Coelho, E.F.; Andrade, C.L.T.; Or, D.; Lopes, L.C.; Souza, C.F. Desempenho de diferentes guias de ondas para uso com o analisador de umidade Trase. Revista Brasileira de Engenharia Agrícola e Ambiental, Campina Grande, v.5, n.1, p.81-87.2001.

Coelho, E.F.; Or, D. A parametric model for two-dimensional water uptake by corn roots under drip irrigation. Soil Science Society of America Journal, Madison, v.60, n. 4, p.10391049.1996.

Conciani, W.; Carneiro, B.J.; Soares, M.M.; Hermann, P.S.P.; Crestana, S. Emprego de TDR com sondas multi-hastes segmentadas para medida de umidade de um perfil de solo. In: Simpósio Nacional de Instrumentação Agropecuária, 1, 1997, São Carlos, Anais... São Carlos: EMBRAPA-CNPDIA, 1997.p.169-173.

Hasted J.B. Aqueous dielectrics. London: Chapman and Hall, 1973. 302p.

Noborio, K. Measurement of soil water content and electrical conductivity by time domain reflectometry: a review. Computers and Electronics in Agriculture, Albuquerque, v.31, n.11, p. 213-237.2001.

Or, D.; Jones, S.B.Thermal and geometrical effects on bulk permittivity of porous mixtures containing bound water. In: Matsura, E.E.; Javarez Junior, A.; Gomes, E.P.; Souza, C.F. (eds.). Aplicações técnicas de TDR na agricultura. Campinas: FEAGRI/UNICAMPI, 2001.p.51-63.

Or, D.; Wraith, J.M. Agricultural and enviromental soil physics. Utah State University. Logan. Utah State University: 1997.218p.

Pepin, S.; Livingston, N.J.; Hook, W.R. Temperature-dependent measurement errors in time domain reflectometry determinations of soil water. Soil Science Society of America Journal. Madison, v.59, n.1, p.38-43. 1993.

Silva, T.S.M.; Coelho, E.F.; Santos, D.B.; Souza, C.F. Avaliação de guias de onda de TDR com diferentes padrões de número e espaçamento entre hastes In. Congresso Brasileiro de Engenharia Agrícola, 31, 2002, Salvador. A engenharia agrícola para o desenvolvimento sustentável água, energia e meio ambiente, Salvador. Resumos...Salvador:SBEA, 2002, CDRom.

Souza, C.F.; Matsura, E.E.; Testezlaf, R. Experiência de laboratório de hidráulica, irrigação e drenagem da Faculdade de Engenharia Agrícola/UNICAMP no uso da técnica da TDR. In: Matsura, E.E.; Javarez Junior,A.; Gomes, E.P.; Souza, C.F. (eds.). Aplicações técnicas de TDR na agricultura. Campinas: FEAGRI/UNICAMPI, 2001.p.147-176.

Topp,G.C.; Davis, J.L.; Annan, A. Electromagnetic determination of soil water content: measurement in coaxial transmission lines. Water Resources Research, Washington, v.16, n.3, p.574-582. 1980.

Wraith, J.; Das, B.S. Monitoring soil water and ionic solute distributions using time domain reflectometry. Soil and Tillage Research, Watkinsville, v.47, p.145-150. 1998.

Wraith, J.M.; Or, D. Temperature effects on soil bulk dielectric permittivity measured by time domain reflectometry: experimental evidence and hypothesis development. Water Resources Research. Washington, v.35, n.2, p.361-369. 1999. 